\title{
Transcriptional profiling distinguishes inner and outer annulus fibrosus from nucleus pulposus in the bovine intervertebral disc
}

\author{
Guus G. H. van den Akker ${ }^{1}$ Marije I. Koenders ${ }^{1} \cdot$ Fons A. J. van de Loo $^{1}$ • \\ Peter L. E. M. van Lent ${ }^{1} \cdot$ Esmeralda Blaney Davidson $^{1} \cdot$ Peter M. van der Kraan $^{1}$
}

Received: 9 September 2016/Revised: 11 May 2017/Accepted: 18 May 2017/Published online: 31 May 2017

(C) The Author(s) 2017. This article is an open access publication

\begin{abstract}
Background Cells in the intervertebral disc have unique phenotypes and marker genes that separate the nucleus pulposus (NP), annulus fibrosus (AF) and articular cartilage (AC) have been identified. Recently, it was shown that phenotypic marker genes exhibit variable expression in humans. In this study, the bovine tail was used to determine the ability of marker genes to distinguish the outer and inner AF from NP tissue and isolated cells.

Methods Bovine tail intervertebral discs from 13 donors were dissected and correct isolation of tissue was confirmed. mRNA was isolated directly from tissue or passage 0 monolayer cells and used for gene expression measurements (qPCR). Conventional marker genes (bAcan, bCollal, bCol2al) and novel marker genes (bAdamts17, bBrachyury/T, bCD24, bCol5al, bCol12al, bFoxf1, bKrt19, bPaxl, bSfrp2) were evaluated.

Results As expected bAcan, bCol2al and bCollal distinguished outer AF from NP tissue, while inner AF and NP could not be discriminated. The NP markers $b T, b C d 24$ and $b$ Krt19 were significantly higher expressed in NP than inner and outer AF tissue. bFoxFl and bPaxl only distinguished IVD tissues from AC. The AF markers $b A$ damts17, bCol5al, bCol12al and bSfrp2 were higher expressed in the outer AF compared with inner AF and NP tissue. Monolayer culturing strongly decreased bAcan, bCol2al, bCD24 and bCol5al expression, while bCollal, $b T, b K r t 19$ and $b S f r p 2$ were not affected.
\end{abstract}

Peter M. van der Kraan

peter.vanderkraan@radboudumc.nl

1 Department of Rheumatology, Radboud University Medical Center, PO Box 9101, 6500 HB Nijmegen, The Netherlands
Conclusion The IVD phenotypic marker genes bT, bKrt19, $b S f r p 2$ and $b$ Coll2al convincingly distinguished NP from outer AF in situ and in vitro.

Keywords Intervertebral disc $\cdot$ Cell biology $\cdot$ Nucleus pulposus · Annulus fibrosus

\section{Introduction}

Low back pain is a large socio-economic problem and is correlated with degeneration of the intervertebral disc (IVD) $[1,2]$. The IVD consists of the central nucleus pulposus (NP) and is surrounded by the ligamentous annulus fibrosus (AF). It is flanked superiorly and anteriorly by the cartilaginous endplates. The AF can be further sub divided in the outer zone (collagen type I rich) and inner zone (proteoglycan and collagen type II rich). Morphologically inner AF cells appear more rounded, while outer AF cells have a fibroblast-like appearance. The IVD is a unique tissue that differs from Articular Cartilage (AC) in embryonic development, nutrient supply, oxygen tension and biochemical composition [3]. Moreover, whole transcriptome analyses of IVD and AC cells revealed distinct gene expression profiles for the $\mathrm{NP}, \mathrm{AF}$ and $\mathrm{AC}$ in rat, canine, bovine and human IVDs [4-7]. These studies began to unravel the NP cell phenotype at the transcriptome level leading to a first consensus paper regarding definition of healthy young NP cells [8].

Previously, we used a subset of genes specifically expressed in the AF or NP to confirm correct isolation of cell populations and identified functional cellular subpopulations in human NP and AF cell lines [9, 10]. In addition, NP specific marker genes are increasingly used as readout to develop stem cell differentiation protocols for 
NP regeneration [11, 12]. However, a recent report described large variation for $\mathrm{NP}$ and $\mathrm{AF}$ marker genes in human cell isolates, which prevented separation of NP and AF tissue based on phenotypic markers [13]. Only PAXI and FOXF1 were confirmed to distinguish NP tissue from $\mathrm{AC}$ and at the protein level NP from AC. Therefore, questions were raised regarding the definition of NP and AF cell phenotypes and the utility of NP marker genes to direct stem cell differentiation.

Acquiring large numbers of non-degenerate human IVD donors is challenging. Therefore, model organisms, such as the cow, are used for in vitro cellular and ex vivo biomechanical studies of the IVD $[14,15]$. The bovine tail IVD represents a good model for the non-degenerate human IVD: the bovine NP retains few notochordal cells and whole transcriptome analyses identified similar phenotypic marker genes as in humans $[6,16,17]$. This study aimed to establish whether IVD phenotypic marker genes, which we previously used to distinguish non-degenerate human NP from AF cells, can be used to describe the cell phenotype in the outer AF, inner AF and NP in situ in the bovine tail IVD and this was compared to passage 0 monolayer cells.

\section{Materials and methods}

\section{Bovine tissue samples and cell isolation}

Bovine tails and metacarpophalangeal joints were obtained from a local slaughterhouse within $3 \mathrm{~h}$ post mortem (Table 1). Muscle was removed and whole IVD were isolated by cutting along the end plates. IVD tissues were

Table 1 Bovine donor characteristics

\begin{tabular}{llll}
\hline IVD donors & Age (years) & AC donors & Age (years) \\
\hline 1 & $<1$ (calf) & 1 & $<1$ (calf) \\
2 & $<1$ (calf) & 2 & $<1$ (calf) \\
3 & $<1$ (calf) & 3 & $<1$ (calf) \\
4 & 2 & 4 & 3.5 \\
5 & 3.5 & 5 & 4.5 \\
6 & 4.5 & 6 & 5 \\
7 & 5 & 7 & 5.5 \\
8 & 7 & 8 & 6 \\
9 & 8.5 & 9 & 6 \\
10 (in vitro) & Unknown & 10 & 8.5 \\
11 (in vitro) & Unknown & & \\
12 (in vitro) & 1 & & \\
13 (in vitro) & 5 & & \\
\hline A & & & \\
\hline
\end{tabular}

A total of nine IVD and ten AC donors were used for in situ gene expression profiling. Four independent donors were used for in vitro gene expression profiling. Age is reported in years if known isolated from the two intact, most proximal tail discs of each donor. Subsequently the NP was dissected and transition zone of approximately $3 \mathrm{~mm}$ was removed and discarded from the remaining tissue. Clearly distinguishable lamellar AF tissue, located closely to the NP, was isolated and termed inner AF. Another $3 \mathrm{~mm}$ of the IVD was removed, discarded and the remaining tissue was termed outer AF (Fig. 1a). Special care was taken not to isolate ligament. For each donor two biological replicates were isolated for RNA expression and two for GAG/DNA measurements. Articular cartilage from the metacarpophalangeal joint was isolated in a standard procedure as described previously [18]. Cells were isolated from four independent donors using overnight digestion with $0.1 \%$ Collagenase Type II (Gibco) in DMEM-F12 (antibiotics). The cells suspension was strained (70 $\mu \mathrm{m}$, Falcon), washed three time with $\mathrm{NaCl}(0.9 \%)$ and plated at 50,000 cells $/ \mathrm{cm}^{2}$ in DMEM-F12 (10\% fetal calf serum, antibiotics). Cells were allowed to adhere for 7 days, washed twice and serum starved for $24 \mathrm{~h}$ prior to sampling.

\section{Histology}

Whole tail IVDs were fixed in phosphate buffered formalin (3.4\%) for $24 \mathrm{~h}$ and dehydrated using an automated tissue processing apparatus (Pathos, Milestone Medical Inc.) followed by embedding in paraffin. Sections of $10 \mu \mathrm{M}$ were cut and stained with Safranin $O$ and counterstained using fast green.

\section{Quantification of sulfated GAG and DNA content}

Tissue pieces were weighed (average $60 \mathrm{mg}$ ) and digested in $1 \mathrm{ml}$ digestion buffer [0.1\% papain, $200 \mathrm{mM}$ $\mathrm{NaPO}_{4}, 100 \mathrm{mM} \mathrm{NaAc}, 5 \mathrm{mM}$ cysteine $\mathrm{HCl}, 10 \mathrm{mM}$ EDTA, pH 6.4 (Sigma)] at $60^{\circ} \mathrm{C}$ overnight. Samples were centrifuged for $10 \mathrm{~min}$ at $13,000 \mathrm{rpm}$ and supernatant was transferred to fresh tubes. Sulfated GAG measurements were done using the dimethylmethylene blue assay as previously described [19]. DNA content was determined using the Picogreen dsDNA assay kit (Quant-it) according to manufacturer's instructions. Diluted samples were compared to a standard of chondroitin sulfate (sGAG) or purified DNA (Picogreen) and the total sGAG or DNA content was calculated per milligram tissue.

\section{mRNA isolation and cDNA synthesis}

Dissected tissue was immediately snap frozen in liquid nitrogen. Prior to mRNA isolation, tissue was homogenized using a dismembrator (B. Braun) and suspended in RLT lysis buffer with mercapto-ethanol (20 mg tissue/ml). 
Fig. 1 Confirmation of isolation of outer $\mathrm{AF}$, inner $\mathrm{AF}$ and NP tissue. a Histological image from the whole bovine tail IVD (sagittal section; left anterior, right posterior) stained with SafraninO/Fast green. The dissection strategy for obtaining outer AF, inner AF and NP tissue is indicated. Bar represents $500 \mu \mathrm{m}$.

b Quantification of sGAG per $\mathrm{mg}$ tissue in nine donors (biological duplicates). c Quantification of DNA per mg tissue in nine donors (biological duplicates). Mean $\pm \mathrm{SD}$,

$* * p<0.01, * * * p<0.001$
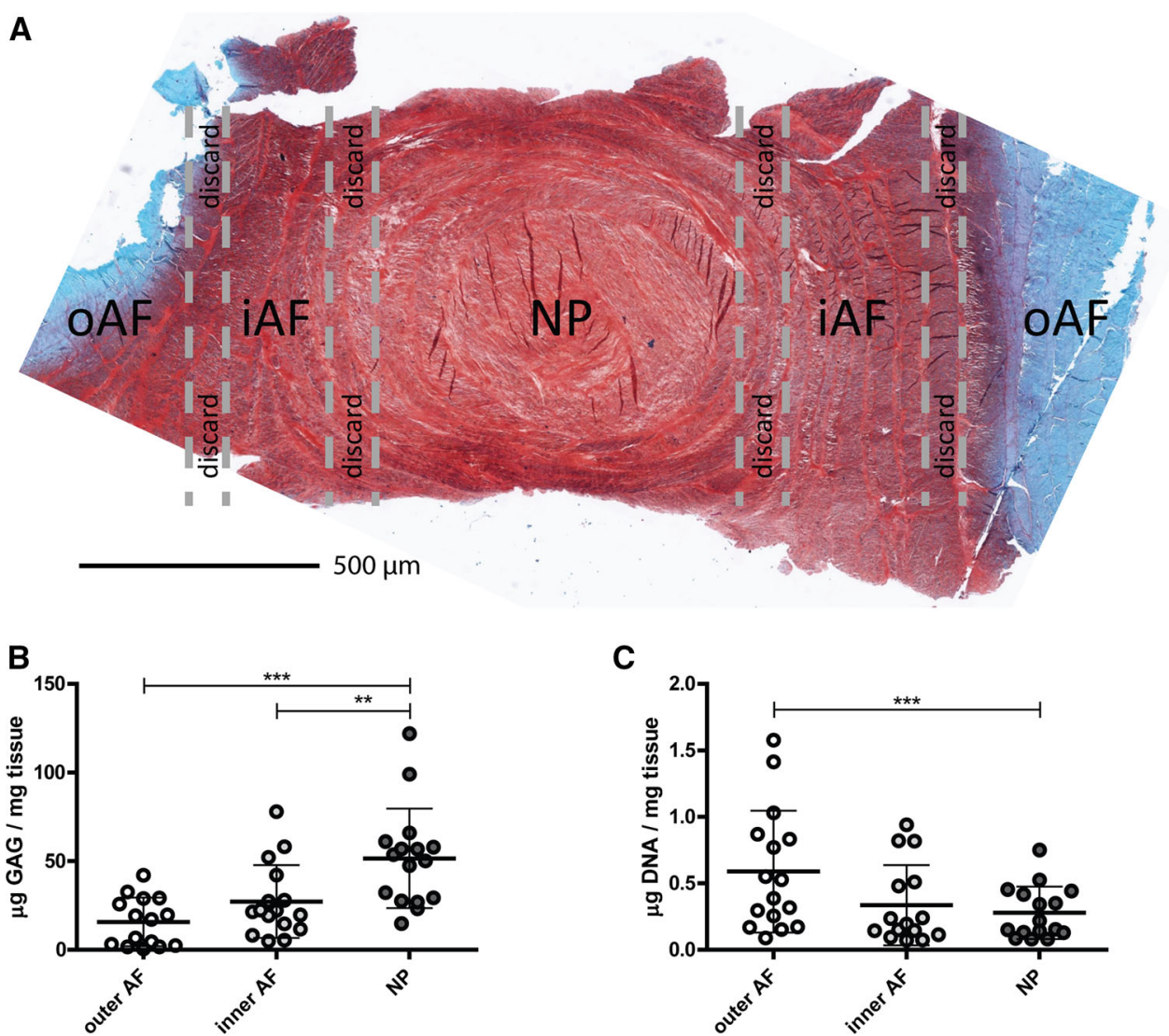

C

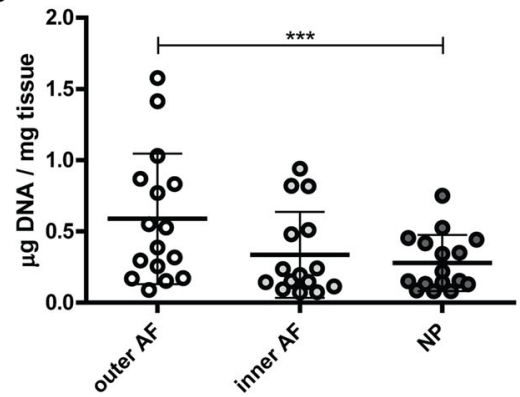

mRNA was isolated using the RNeasy fibrous tissue kit (Qiagen) according to manufacturer's instruction. Next, $8 \mu \mathrm{l}$ of each sample was treated with DNAse (LifeTechnologies) and the mRNA reverse transcribed using an oligo dT primer as previously described [18]. The obtained cDNA was diluted $10 \times$ in RNAse free water and used for gene expression analyses. Monolayer samples were isolated with the RNeasy kit (Qiagen) and $500 \mathrm{ng}$ mRNA was used for cDNA synthesis.

\section{Gene expression analyses}

Primer sets were designed with primer blast and tested for linear amplification on a standard of cultured bovine IVD cell cDNA. Primer sequences are listed in Table 2.

Real-time quantitative polymerase chain reaction (PCR) was performed using a StepOnePlus sequence detection system (Applied Biosystems) and gene expression was normalized to the average of two housekeeper genes (bGapdh and bRps14) using the $-\Delta C_{\mathrm{t}}$ method. Subsequently all genes were expressed relative to the outer $\mathrm{AF}$ in situ $\left(-\Delta \Delta C_{\mathrm{t}}\right)$. The original $-\Delta C_{\mathrm{t}}$ values for the outer $\mathrm{AF}$ are provided in Table 2 .

\section{Statistics}

Statistical significance between two groups was assessed using a two sided $t$ test with Welsh's correction. Figures were generated and statistical analyses performed using Graphpad Prism V5.0.

\section{Results}

Prior to sample isolation, histological analysis of bovine tail IVDs was performed to determine the relative size of inner and outer AF regions (Fig. 1a). Outer AF, inner AF and NP tissue from nine bovine donors was isolated and correct isolation of the tissues was assessed by determining sGAG and DNA content. NP tissue had a significantly higher GAG content then inner AF or outer AF tissue (Fig. 1b). Inversely, the outer AF contained significantly more DNA then NP tissue (Fig. 1c). This is in agreement with known proteoglycan and cell content of NP and AF tissue confirming correct tissue isolation [20, 21].

Subsequently, the expression of two characteristic chondrocyte genes Aggrecan (bAcan) and Collagen type II 
Table 2 Bovine primer sets used for RT-qPCR measurements

\begin{tabular}{|c|c|c|c|c|}
\hline Gene & Ref seq & Forward primer & Reverse primer & $\mathrm{oAF}\left(-\Delta C_{\mathrm{t}}\right)$ \\
\hline bAcan & NM_173981.2 & TGAAACCACCTCCACCTTCCATGA & TCAAAGGCAGTGGTTGACTCTCCA & +2.5 \\
\hline$b$ Col2al & NM_001001135.2 & TGATCGAGTACCGGTCACAGAA & CCATGGGTGCAATGTCAATG & +4.1 \\
\hline bCollal & NM_174520.2 & CTGGGTACCACCGTTGATAGTTT & AGTCAAGAACTGGTACAGAAATTCCAA & +1.0 \\
\hline$b T$ & NM_001192985.1 & CACACGGCTGCGAAAGGTA & TGAACTGTCGGAATAGGTTGGA & -13.6 \\
\hline$b C d 24$ & XM_002690126.1 & TGCTCTTACCTACGCAGACTTAC & GCTGTTGACTGCAGAGTACCA & -8.7 \\
\hline bKrt19 & NM_001015600.1 & GACCTGCGGGACCAGATTCTC & GTCAGCCTCCACACTCATGC & -8.6 \\
\hline bFoxf1 & XM_003583371.1 & CGGCCAGCGAGTTCATGTTT & CGAGCCCGTTCATCATGCTAT & -4.8 \\
\hline bPaxl & XM_015474037.1 & GAAGACTGGGCGGGAGTGAA & AGGCCGACTGCGTGTATTTA & -3.2 \\
\hline bAdamts 17 & XM_010816917.1 & TCTGCAGAAACATGGAGCATCT & GGAGGGTCCAGTTTGGTCTT & -7.4 \\
\hline bSfrp2 & NM_001034393.1 & CAGGACAACGACCTTTGCAT & TCACATACCTTTGGAGCTTCCT & -6.6 \\
\hline bCol5al & XM_002691722.3 & AGATGGCAAGTGGCACAGAAT & GGTCCAGGAACTTGGTTGTCT & -0.2 \\
\hline$b$ Col12al & NM_001206497 & ACCGGCTACACTGTGACCTA & TCCAGGCGCATCTCTTTGG & -1.1 \\
\hline bGapdh & NM_001034034.2 & CACCCACGGCAAGTTCAAC & TCTCGCTCCTGGAAGATGGT & NA \\
\hline bRps14 & NM_001077830.2 & CATCACTGCCCTCCACATCA & TTCCAATCCGCCCAATCTTCA & NA \\
\hline
\end{tabular}

Gene symbols, reference sequence of the transcript and the $5^{\prime}-3^{\prime}$ primer sequences. The minus delta $C_{\mathrm{t}}$ values for each gene in outer $\mathrm{AF}$ tissue, which was used for minus $\Delta \Delta C_{\mathrm{t}}$ calculations, is provided in the last column

$(b$ Col2al)) was determined. Both genes were significantly higher expressed in the inner AF (bAcan 3.4 fold; $b$ Col2al 6.3 fold) and NP (bAcan 3.2 fold; bCol2al 7.1 fold) compared to outer AF (Fig. 2a). No difference was found between inner AF and NP. To asses NP differences with AC we obtained cDNA from ten independent donors with a comparable age distribution. AC showed significantly lower expression of bAcan (6.8 fold) and equal bCol2al expression compared with the NP. This is consistent with reported differences in matrix composition of $\mathrm{AC}$ and $\mathrm{NP}$ tissue [22]. As a positive marker gene for the AF we determined Collagen type I (bCollal) expression. Collal was significantly higher expressed in the outer AF (47.0 fold) over inner $\mathrm{AF}$ and 56.8 fold compared to NP (Fig. 2c). AC showed significantly lower bCollal expression than the NP (Fig. 2c). In summary, conventional marker genes allowed the discrimination of outer AF from $\mathrm{NP}$ and AC tissue. However, a distinction between the NP and inner AF could not be made. Monolayer culturing of isolated IVD cells (passage 0) led to an average 22 fold reduction in bAcan and 115 fold reduction in bCol2al expression, while bCollal was unaffected by in vitro culturing (Fig. 2a-c, right panel). Nevertheless, differential expression between NP, iAF and oAF cells was maintained for all three genes in vitro.

Previously we used six NP specific marker genes to discriminate cultured human NP from AF cells [10]. We hypothesized that these genes would be able to distinguish the NP from inner AF tissue. Five human NP marker genes were evaluated in bovine tissue: Brachyury/T $(b T)$, Keratin 19 (bKrt19), Cluster of differentiation 24 (bCd24),
Forkhead box transcription factor 1 (bFoxf 1$)$ and Paired box 1 (bPaxl). Carbonic anhydrase XII (bCa12) was not reliably detected, despite the use of multiple primer sets. We detected $b T$ mRNA in nearly all NP tissue samples, while it was hardly detectable in $\mathrm{AF}$ and $\mathrm{AC}$ samples (Fig. 3a). In 3/18 inner AF and 8/18 outer AF tissue samples $b T$ mRNA was detected albeit with a 104 fold lower expression than NP, which approached the detection limit. bKrt19 was significantly higher expressed in NP tissue compared with the AF (inner 5.3 fold; outer 5.8 fold) and AC (73 fold; Fig. 3c). bKrt19 was not detectable in 14/36 $\mathrm{AF}$ and 3/9 AC samples. $b C d 24$ was detected in almost all samples with a significantly higher expression in NP compared to inner (3.4 fold) and outer AF (4.3 fold; Fig. 3b). AC had a similar $b C d 24$ expression compared to the NP and $b C d 24$ is, therefore, not specific for the IVD. $b T$ expression was not affected by monolayer culture (Fig. 3a, right panel), while $b \operatorname{Krt} 19$ appeared reduced in monolayer culture, this was only significant in the oAF (Fig. 3b, right panel). Importantly, differential expression of bKrt19 was maintained in vitro. $b C D 24$ was decreased by $15-47$ fold in all cell types and differential expression was lost (Fig. 3c, right panel). bFoxfl and bPaxl were detected in nearly all IVD samples and showed no differential expression within the bovine tail IVD (Fig. 4a, b). These genes were originally identified as NP markers in comparison with AC [7]. Indeed AC showed 90.5 (bFoxfl) to 388.0 fold (bPaxl) lower expression levels. In vitro culturing of IVD cells led to a two- to fourfold decrease in bFoxfl and bPaxl expression that was not always statistically significant (Fig. 4, right panels). In conclusion, $b T$ appears to be the 
Fig. 2 The conventional IVD marker genes Acan, Col2a1 and Colla1 discriminate outer $\mathrm{AF}$ from inner AF or NP. Left panels gene expression measurements on nine IVD donors (biological duplicates) and ten AC donors (single measurement per donor) for a bAcan, b bCol2al and c $b$ Colla1. Right panels gene expression measurements for indicated genes in four bovine cell isolates at passage 0 (biological triplicates). ${ }^{\#} p<0.05$ compared to in situ expression in the same tissue. Gene expression was normalized to the in situ expression in the outer $\mathrm{AF}$ $\left(-\Delta \Delta C_{\mathrm{t}}\right)$. Original $-\Delta C_{\mathrm{t}}$ values in the oAF can be found in Table 1. Mean $\pm \mathrm{SD}$, $* * p<0.01, * * * p<0.001$
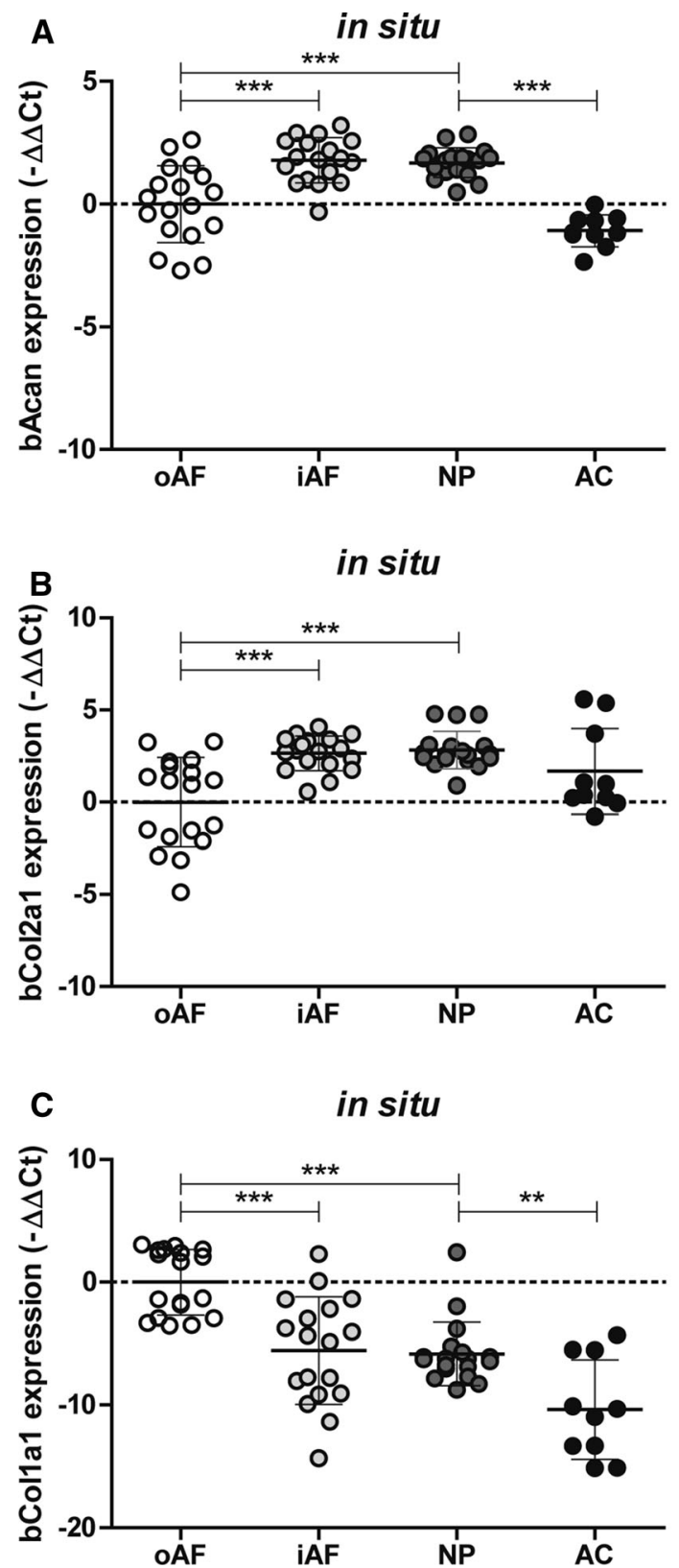

in vitro

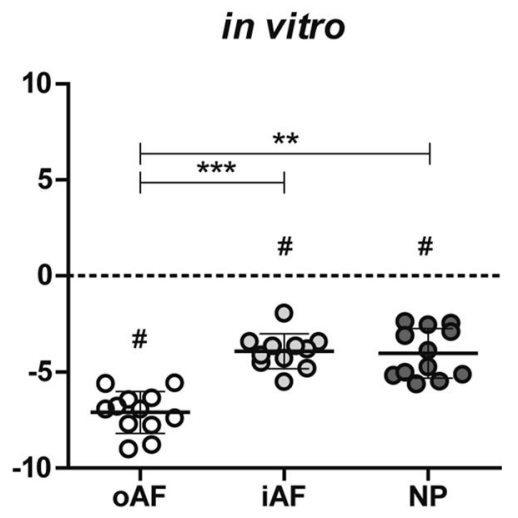

in vitro

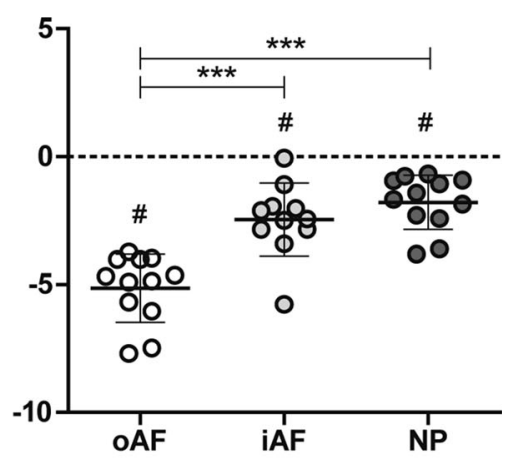

in vitro

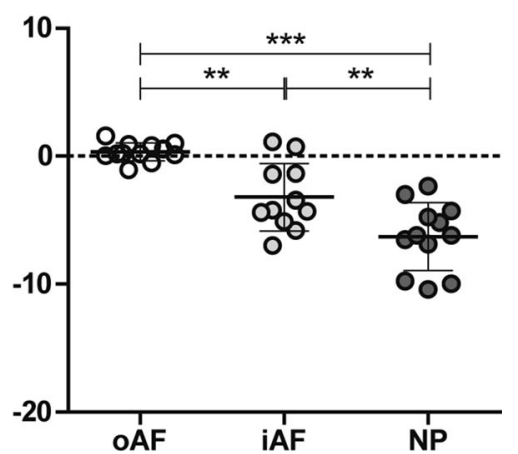

most sensitive NP marker gene; whereas the significant expression differences for $b K r t 19$ and $b C d 24$ compared to the outer AF were in the same range as conventional marker genes. In addition, we find that bFoxfl and bPax 1 are not differentially expressed in the bovine tail IVD.

ADAMTS17, SFRP2, COL5A1 and COL12A1 were previously identified as (outer) AF markers compared to NP cells [9]. To test if these marker genes distinguish inner from outer AF tissue, they were evaluated in the bovine tail IVD. bAdamts 17 was significantly higher expressed in outer AF tissue compared to inner AF (2.1 fold) and NP (2 fold) with relatively low inter-donor variation (Fig. 5a). SFRP2 was the most differentially expressed AF marker gene in an earlier in vitro study [9].
In agreement with this, we found that bSfrp2 was significantly higher expressed in the outer AF compared with the inner AF (7.9 fold) and NP (29.0 fold) (Fig. 5b). Moreover, bSfrp2 expression was detected in all outer AF samples, while it was not detectable in 10/18 inner AF, in 13/18 NP and 8/10 AC samples (Fig. 5b). Col5al showed a similar significant 2.3 fold expression difference as bAdamts17 (Fig. 5c). Coll2al had the strongest differential expression in the outer AF compared to the inner AF (15.5 fold) and was significantly lower expressed in the NP (24.3 fold; Fig. 5d). It was expected that NP and AC tissue would be similar (cf. Fig. 2), however, AC had significantly higher expression of bCol5al and $b$ Coll2al compared to NP tissue and this did not differ from the 
Fig. 3 The NP marker genes $b T, b C d 24$ and bKrt19 are able to distinguish inner AF from NP tissue and cells. Left panels gene expression measurements on nine IVD donors (biological duplicates) and ten AC donors (single measurement per donor) for a $b T, \mathbf{b}, b C d 24, \mathbf{c}, b K r t 19$. Right panels gene expression measurements for indicated genes in four bovine cell isolates at passage 0 (biological triplicates). ${ }^{\#} p<0.05$ compared to in situ expression in the same tissue. Gene expression was normalized to the in situ expression in the outer $\mathrm{AF}$ $\left(-\Delta \Delta C_{\mathrm{t}}\right)$. Original $-\Delta C_{\mathrm{t}}$ values in the oAF can be found in Table 1. Mean $\pm \mathrm{SD}$, ${ }^{*} p<0.05,{ }^{* *} p<0.01$, $* * * p<0.001, N D$ not detected
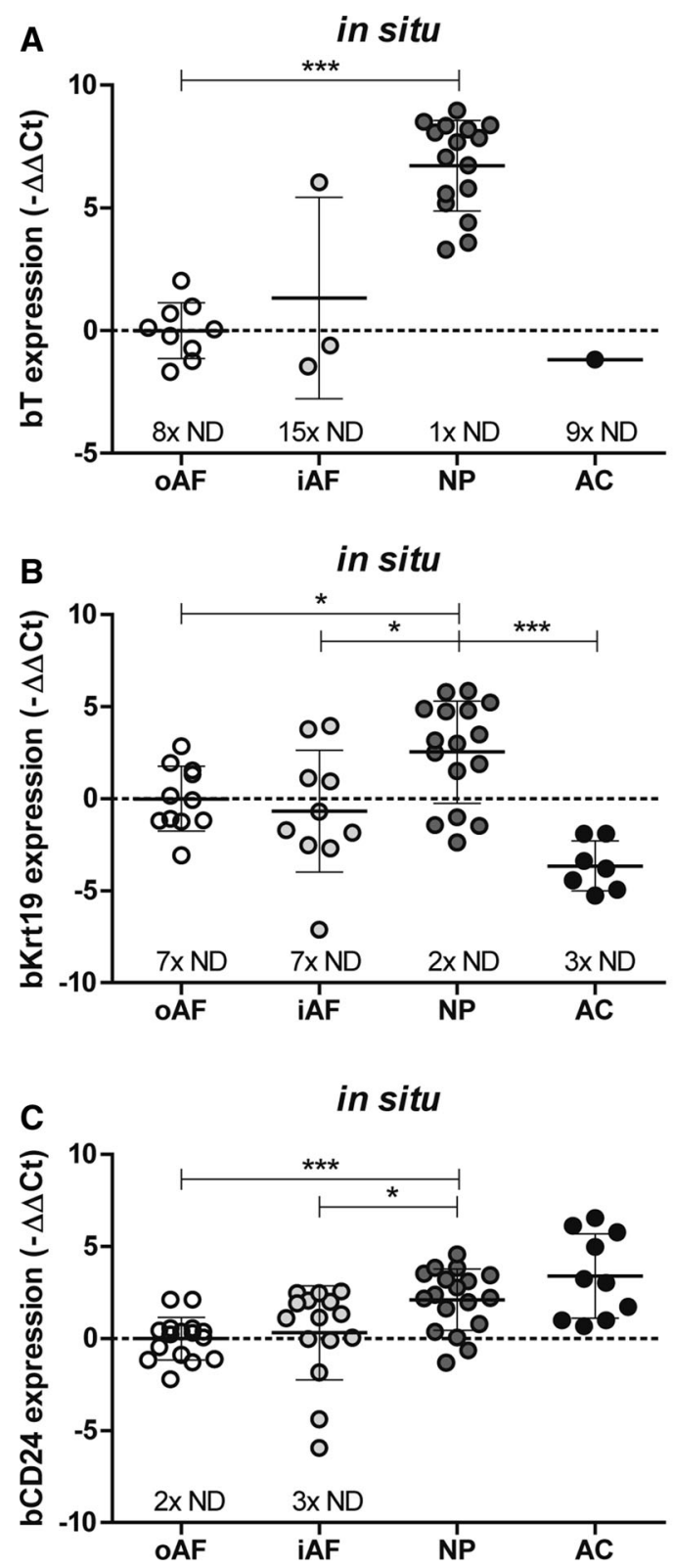

in vitro

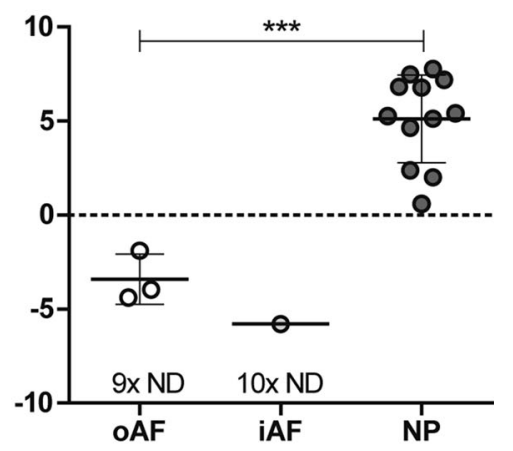

in vitro

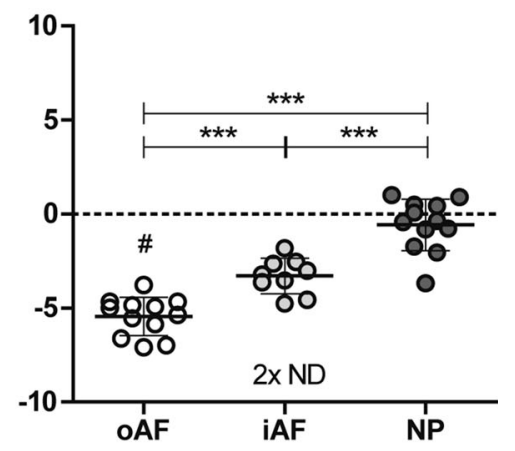

in vitro

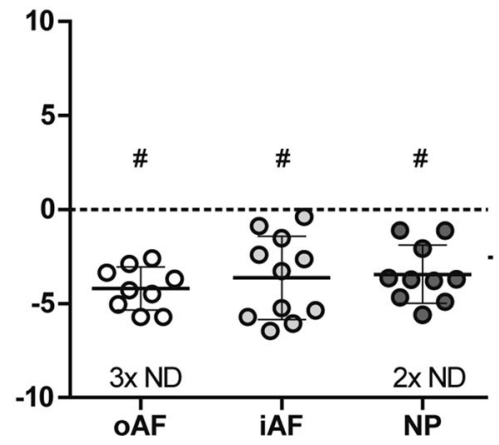

outer AF (Fig. 5c, d). In vitro culturing negated differential expression of bAdamts17, while bSfrp2 expression was maintained in the oAF (Fig. 5a, b). A significant reduction of $b$ Col5al expression (average of 7.4 fold) was observed when compared to in situ. Finally, bCol12al was slightly decreased in the oAF (1.4 fold) and increased in iAF (6.0 fold) and (2.3 fold) NP cells (Fig. 5c, d). Nevertheless, differential expression of $b$ Col5al and $b$ Col12al was maintained in vitro. In summary, all AF marker genes distinguished outer AF tissue from inner $\mathrm{AF}$ and NP tissue. However, bSfrp2 and bCol12al showed higher fold expression differences than conventional marker genes. Differential expression of bSfrp2, bCol5al and $b$ Coll2al was maintained in vitro.

\section{Discussion}

In this study, we aimed to establish qualitative differences in $\mathrm{NP}$ and AF specific marker genes in the bovine tail IVD, as a model for the non-degenerate healthy human IVD, and compared them to conventional marker genes. In addition, we evaluated the ability of these marker genes to distinguish inner AF from NP or outer AF and finally the effect of cell isolation and subsequent monolayer culture at passage 0 . Our main research findings are summarized in Table 3.

A number of our selected marker genes (T, KRT19, $C D 24)$ are recommended for young healthy NP cells [8]. Our study demonstrates that NP marker genes $b T, b K r t 19$, $b C d 24$ can be used over a broad age range to characterize 
Fig. 4 The putative NP marker genes bFoxf1 and bPax1 are not able to distinguish NP from $\mathrm{AF}$ tissue or cells. Left panels gene expression measurements on nine IVD donors (biological duplicates) and ten AC donors (single measurement per donor) for $\mathbf{a}$ bFoxF 1 and $\mathbf{b}$ bPaxl. Right panels gene expression measurements for indicated genes in four bovine cell isolates at passage 0 (biological triplicates). ${ }^{\#} p<0.05$ compared to in situ expression in the same tissue. Gene expression was normalized to the in situ expression in the outer $\mathrm{AF}$ $\left(-\Delta \Delta C_{\mathrm{t}}\right)$. Original $-\Delta C_{\mathrm{t}}$ values in the oAF can be found in Table 1. Mean $\pm \mathrm{SD}$, $* * * p<0.001, N D$ not detected
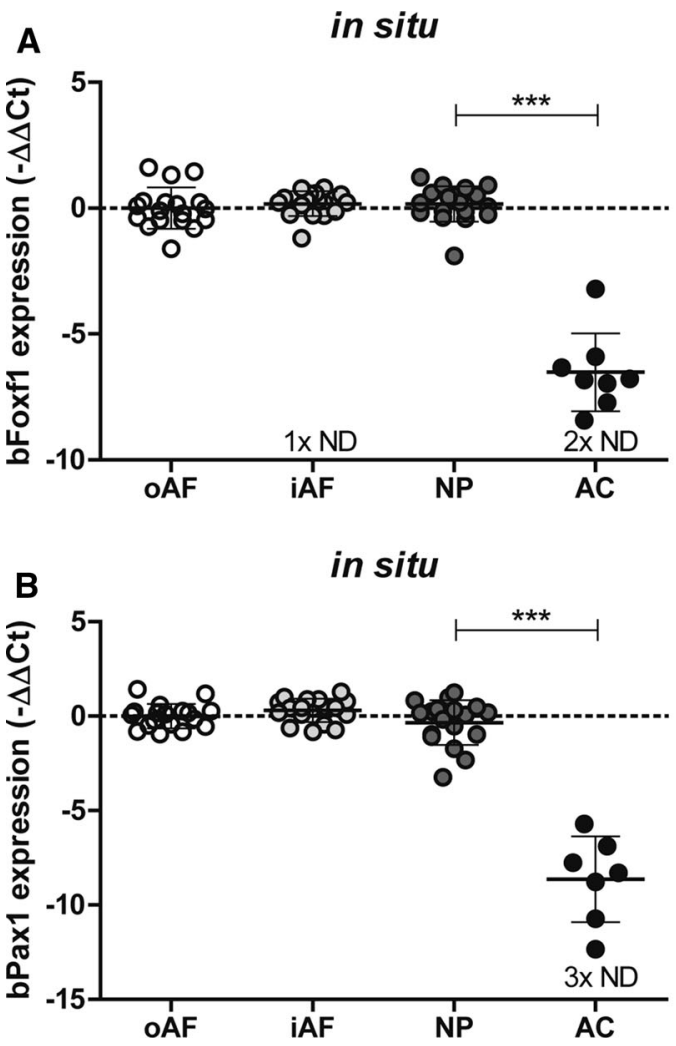

in vitro

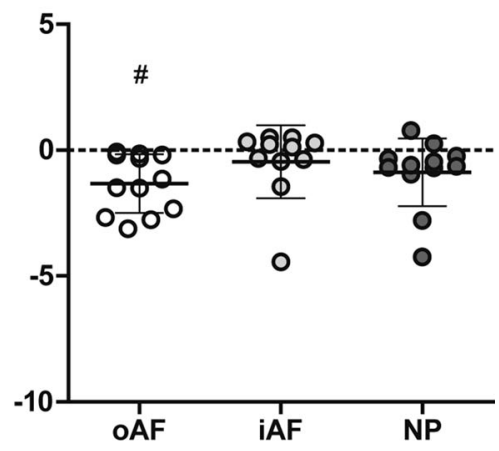

in vitro

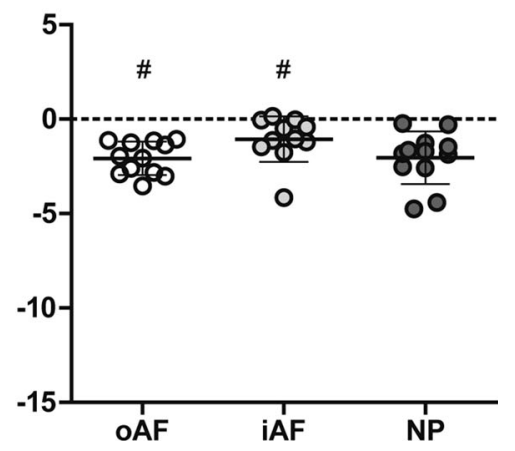

bovine NP tissue. However, differential expression of $b C d 24$ was small in situ and expression was strongly decreased by in vitro culturing. We found that $b T$ and $b K r t 19$ are highly sensitive NP marker genes that are in contrast to bAcan and bCol2al hardly affected by in vitro culturing at passage 0 . Compared to conventional marker genes $b T$ and $b K R T 19$ were rather difficult to detect, exemplified by the large number of non-detectable samples in tissues with low expression. In agreement with other studies, we found that the NP/IVD can be distinguished from AC by high expression of bPaxl and bFoxfl [7, 13]. These genes exhibited the highest differential expression in our study. In comparison to a bovine micro-array transcriptional profiling study using three donors [6], we also found differential expression of $b K r t 19$ between NP and AC tissue, but not for bFoxfl between NP and AF tissue and $b C D 24$ between NP and AC tissue. The lack of detectable $C A 12$ expression in the bovine tail IVD is supported by literature [6].

The cow is considered to have a similar IVD as humans, with retention of few notochordal cells $[16,23]$. However, the exact relationship between cow age and NC content is not well established. Due to the broad donor age range used in this study (1-8.5 years old), NP tissue may contain varying amounts of notochordal cells (NC). It has been suggested that up to $10 \%$ of bovine NP cells per tail IVD are NC, based on size exclusion and/or Keratin 8 expression [17, 24]. The latter study used 18-24 month old bovine donors, while the former study did not report donor age. This is in contradiction with an earlier study where no evidence for NC was found in 18-24 month old cow tail IVDs [25]. Age separation of our dataset did not reveal a reduction in $b T$ or $b K r t 19$ in bovine donors aged above 24 or 48 months. In support of our data, it was recently shown by RNA in situ hybridization that NP and transition zone cells, which did not have a NC morphology, express bKrt19 [26]. Moreover, we previously found that Brachyury/T mRNA and protein levels do not necessarily match in mature human NP cells [10]. A detailed age range should be performed on the histological level to determine the rate of NC depletion in the bovine tail IVD.

The AF marker genes ADAMTS17, SFRP2, COL5A1 and COL12A1, previously used for cultured NP and AF cells, could be used to distinguish bovine AF from NP tissue [9]. Few studies have addressed inner and outer AF cell phenotypes [27-29]. In our study, bAcan, bCol2al, bCD24, bColla1, bAdamts17, bSfrp2, bCol5al and bCol12al did not distinguish inner AF from NP tissue. In vitro differences between NP and inner AF cells could be detected for $b$ Collal, $b$ Col5al and boll2al. As bCollal expression was not affected by in vitro culturing, this might indicate that the differential expression for NP and iAF is caused by the lower number of donors compared to in situ. On the contrary $b$ Col5al and $b$ Coll $2 a l$ were affected by in vitro culturing and 
Fig. 5 AF marker genes separate outer AF from inner AF or NP tissue and do not discriminate isolated cells. Left panels gene expression measurements on nine IVD donors (biological duplicates) and ten AC donors (single measurement per donor) for a bAdamts17, b bSfrp2, c bCol5a1, d Col12a1. Right panels gene expression measurements for indicated genes in four bovine cell isolates at passage 0 (biological triplicates). ${ }^{*} p<0.05$ compared to in situ expression in the same tissue. Gene expression was normalized to the in situ expression in the outer $\mathrm{AF}$ $\left(-\Delta \Delta C_{\mathrm{t}}\right)$. Original $-\Delta C_{\mathrm{t}}$ values in the oAF can be found in

Table 1. Mean \pm SD,

$* p<0.05, * * p<0.01$,

$* * * p<0.001, N D$ not detected

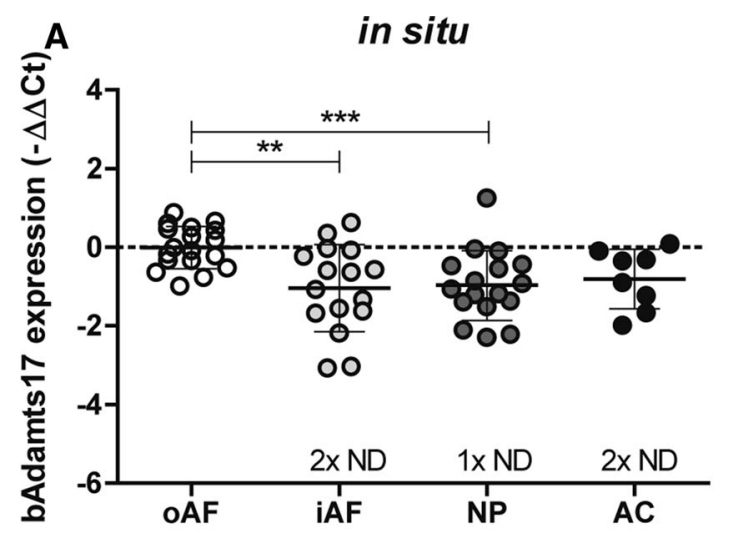

in vitro
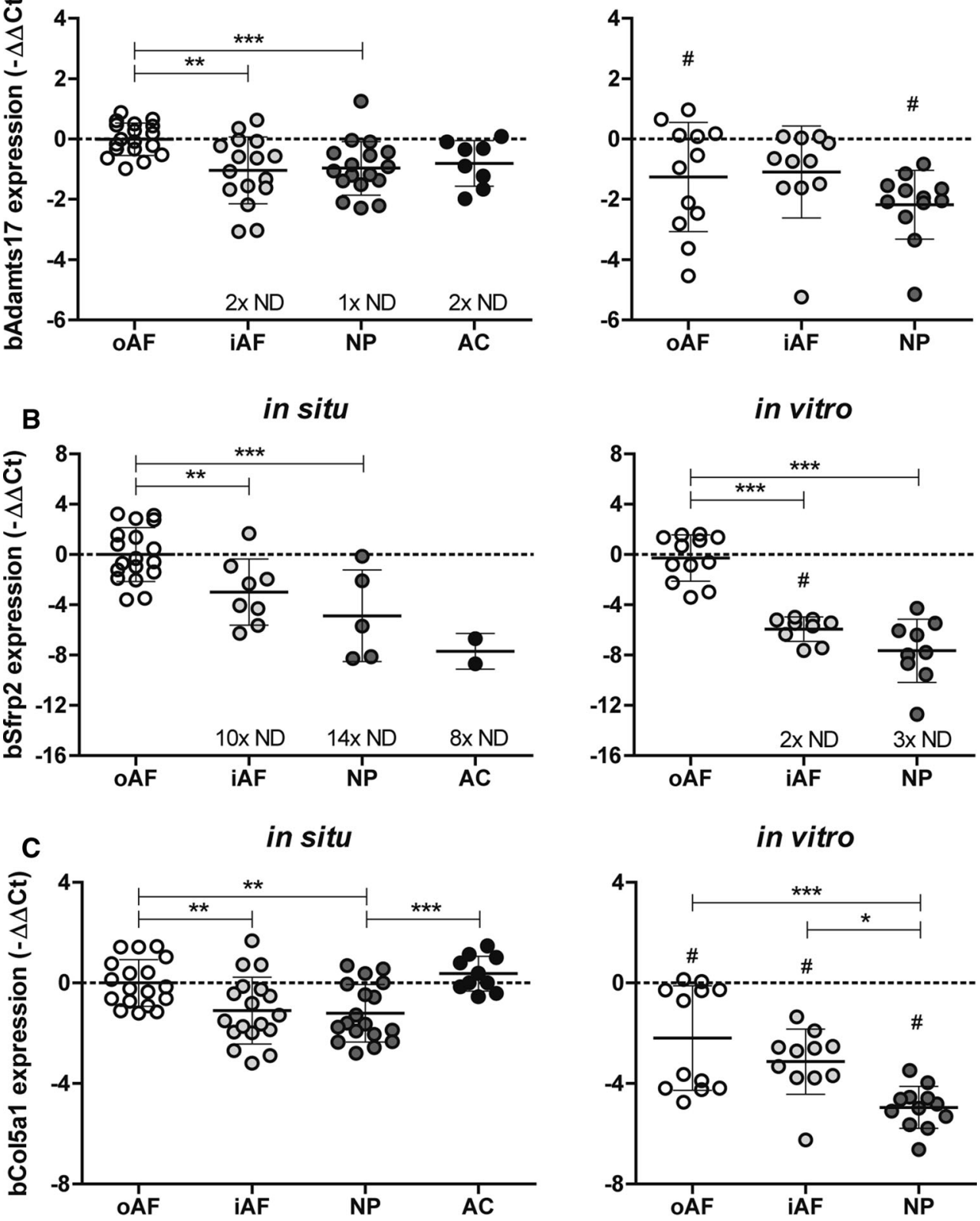

in vitro

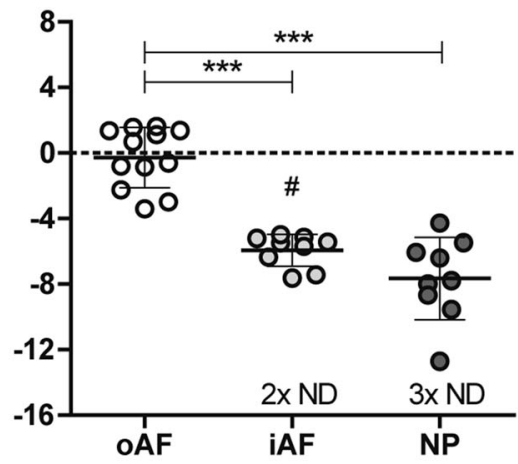

in vitro

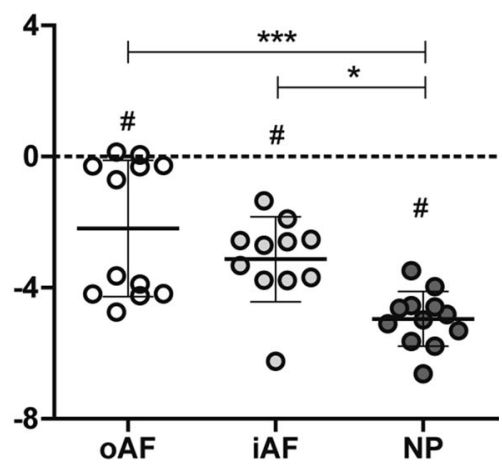

in vitro

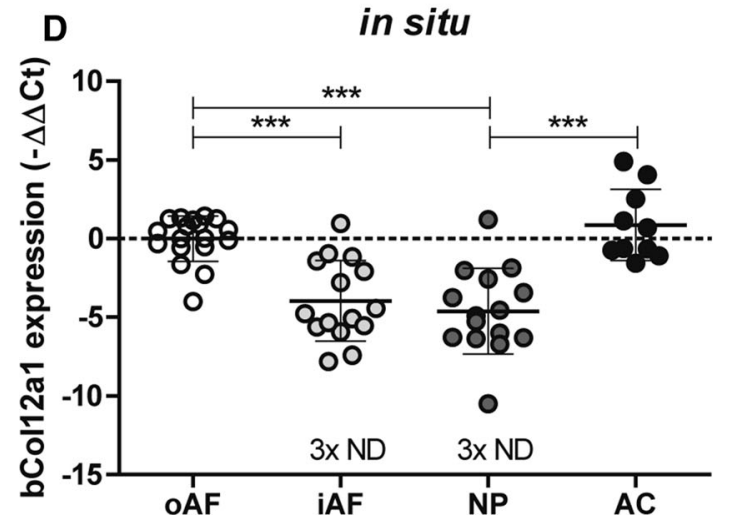

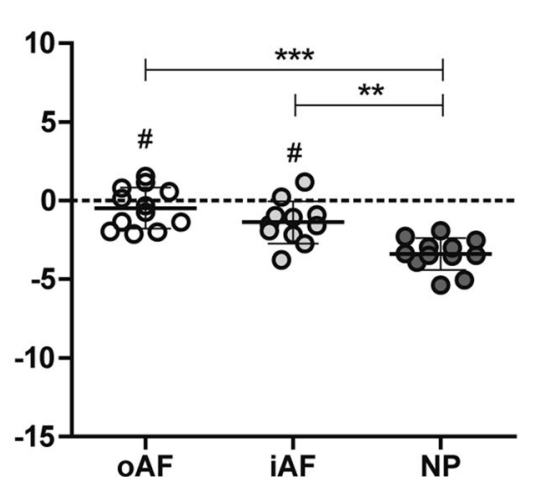


Table 3 Summary of transcriptional profiling of the bovine tail IVD in situ and in vitro

\begin{tabular}{llllllll}
\hline & bAcan & bCol2al & bCollal & $b T$ & bKrt19 & bSfrp2 & bCol12al \\
\hline NP tissue & ++ & ++ & + & +++ & ++ & - & + \\
Inner AF tissue & ++ & ++ & + & - & $+/-$ & $+/-$ & + \\
Outer AF tissue & + & + & +++ & - & $+/-$ & +++ & +++ \\
In vitro marker & Yes & Yes & Yes & Yes & Yes & Yes & Yes \\
In vitro regulation & $\downarrow \downarrow \downarrow$ & $\downarrow \downarrow \downarrow$ & $=$ & $=$ & $=$ & $=$ & $=$ \\
\hline
\end{tabular}

In situ expression: - , not detectable; $+/-$, no/very little expression; + , expression detected in (nearly) all samples; ++ , moderate expression; +++ , high expression. In vitro marker: + , yes; $+/-$, unclear; - , no. In vitro regulation: $=$, no change; $\downarrow \downarrow \downarrow$, strong down regulation this effect appeared to differ between NP and iAF cells. Overall, inner AF tissue and cells appeared to share more characteristics with the NP then the outer AF. Notable exceptions are $b T, b K r t 19$ (in situ, in vitro), and $b C d 24$ (in situ). We here identify $b S f r p 2$ and $b$ Coll2al as sensitive outer AF marker genes when compared with inner AF or NP tissue. Although bAdamts 17 and Col5al are significantly higher expressed in the outer AF, the difference in expression is modest. In contrast to NP marker genes, all four AF marker genes were altered by some degree through in vitro culturing and led to the loss of differential expression for bAdamts 17 , $b$ Col5al and bColl2al between outer AF and inner AF. A positive marker gene for inner AF cells compared to NP or outer AF is currently lacking. This would be extremely useful to exclude that inner AF cells are present in NP or outer AF cell isolations and vice versa.

Finally, an unexpected overlap was found between outer $\mathrm{AF}$ and AC tissue for $b$ Col2al, bCol5al and $b$ Coll2al expression in situ. This may be explained by the fact that AF and NP tissue together fulfills a similar biomechanical function as $\mathrm{AC}$ and that, therefore, structural components from both AF and NP tissue can be found in AC.

NP specific marker genes (compared to AC or AF) are increasingly used to evaluate stem cell differentiation towards an NP phenotype $[12,30]$. It is preferable to use unique NP marker genes that are not expressed in $\mathrm{AF}$ and $\mathrm{AC}$ tissue. However, an ideal NP marker gene set has not yet been identified. Based on our results $b T$ and $b K r t 19$ would be more suitable than $b C d 24, b P a x l$ or $b F o x f 1$, as the latter genes are strongly expressed in outer AF (bPaxl, bFoxfl) and AC tissue (bCd24). To exclude a mixed NP/ $\mathrm{AF}$ phenotype reporting of AF marker gene expression in NP stem cell differentiation assays might be crucial to further improve NP stem cell differentiation assays.

\section{Conclusion}

We aimed to better characterize the outer $\mathrm{AF}$, inner $\mathrm{AF}$ and NP cell phenotype in the bovine tail IVD using a candidate approach. Initial characterization employing conventional marker genes bAcan, bCol2al and bCollal distinguished outer AF from NP, yet failed to distinguish inner AF from NP tissue. We identified $b T, b K r t 19$ as sensitive markers for NP tissue or isolated cells compared with the inner and outer AF. bSfrp 2 and $b$ Coll2al were identified as highly sensitive markers for outer AF tissue compared with inner $\mathrm{AF}$ and NP. The IVD was discriminated from AC by high bPaxl and Foxfl expression. In addition bAcan, bCollal, $b T$ and $b K r t 19$ were higher expressed in NP compared with $\mathrm{AC}$ tissue. Positive selection markers for the inner AF were not identified. IVD phenotypic marker genes represent valuable tools to define cell phenotypes and may elucidate cellular changes that lead to disc degeneration and ultimately low back pain.

Acknowledgements This research was supported by the UK regenerative medicine program SMART STEP. We would like to thank A. Rorije and M. P. Bakx for help with data collection.

\section{Compliance with ethical standards}

Conflict of interest The authors declare that they have no competing interests.

Open Access This article is distributed under the terms of the Creative Commons Attribution 4.0 International License (http://crea tivecommons.org/licenses/by/4.0/), which permits unrestricted use, distribution, and reproduction in any medium, provided you give appropriate credit to the original author(s) and the source, provide a link to the Creative Commons license, and indicate if changes were made.

\section{References}

1. Maniadakis N, Gray A (2000) The economic burden of back pain in the UK. Pain 84(1):95-103

2. Luoma K, Riihimäki H, Luukkonen R, Raininko R, ViikariJuntura E, Lamminen A (2000) Low back pain in relation to lumbar disc degeneration. Spine 25(4):487-492

3. Pattappa G, Li Z, Peroglio M, Wismer N, Alini M, Grad S (2012) Diversity of intervertebral disc cells: phenotype and function. J Anat 221(6):480-496

4. Lee CR, Sakai D, Nakai T, Toyama K, Mochida J, Alini M, Grad S (2007) A phenotypic comparison of intervertebral disc and articular cartilage cells in the rat. Eur Spine J 12:2174-2185 
5. Sakai D, Nakai T, Mochida J, Alini M, Grad S (2009) Differential phenotype of intervertebral disc cells: microarray and immunohistochemical analysis of canine nucleus pulposus and anulus fibrosus. Spine 34(14):1448-1456

6. Minogue BM, Richardson SM, Zeef LAH, Freemont AJ, Hoyland JA (2010) Transcriptional profiling of bovine intervertebral disc cells: implications for identification of normal and degenerate human intervertebral disc cell phenotypes. Arthritis Res Ther 12(1):R22

7. Minogue BM, Richardson SM, Zeef LAH, Freemont AJ, Hoyland JA (2010) Characterization of the human nucleus pulposus cell phenotype and evaluation of novel marker gene expression to define adult stem cell differentiation. Arthritis Rheum 62(12):3695-3705

8. Risbud MV, Schoepflin ZR, Mwale F, Kandel RA, Grad S, Iatridis JC, Sakai D, Hoyland JA (2015) Defining the phenotype of young healthy nucleus pulposus cells: recommendations of the Spine Research Interest Group at the 2014 annual ORS meeting. J Orthop Res 33(3):283-293

9. van den Akker GGH, Surtel DAM, Cremers A, Rodrigues-Pinto R, Richardson SM, Hoyland JA, van Rhijn LW, Welting TJM, Voncken JW (2014) Novel immortal human cell lines reveal subpopulations in the nucleus pulposus. Arthritis Res Ther 16(3):R135

10. van den Akker GG, Surtel DA, Cremers A, Richardson SM, Hoyland JA, van Rhijn LW, Voncken JW, Welting TJ (2016) Novel immortal cell lines support cellular heterogeneity in the human annulus fibrosus. PLoS One 11(1):e0144497

11. Rodrigues-Pinto R, Richardson S, Hoyland J (2014) An understanding of intervertebral disc development, maturation and cell phenotype provides clues to direct cell-based tissue regeneration therapies for disc degeneration. Eur Spine J 23(9):1803-1814

12. Colombier P, Clouet J, Boyer C, Ruel M, Bonin G, Lesoeur J, Moreau A, Fellah B-H, Weiss P, Lescaudron L, Camus A, Guicheux J (2016) TGF- $\beta 1$ and GDF5 act synergistically to drive the differentiation of human adipose stromal cells toward nucleus pulposus-like cells. Stem Cells 34(3):653-667

13. Thorpe AA, Binch AL, Creemers LB, Sammon C, Le Maitre CL (2016) Nucleus pulposus phenotypic markers to determine stem cell differentiation: fact or fiction? Oncotarget 7(3):2189-2200

14. Walter BA, Korecki CL, Purmessur D, Roughley PJ, Michalek AJ, Iatridis JC (2011) Complex loading affects intervertebral disc mechanics and biology. Osteoarthr Cartil 19(8):1011-1018

15. Chan SCW, Walser J, Käppeli P, Shamsollahi MJ, Ferguson SJ, Gantenbein-Ritter B (2013) Region specific response of intervertebral disc cells to complex dynamic loading: an organ culture study using a dynamic torsion-compression bioreactor. PLoS One 8(8): 72489

16. Demers CN, Antoniou J, Mwale F (2004) Value and limitations of using the bovine tail as a model for the human lumbar spine. Spine 29(24):2793-2799

17. Saggese T, Redey P, McGlashan SR (2015) Same-species phenotypic comparison of notochordal and mature nucleus pulposus cells. Eur Spine J 24(9):1976-1985
18. van Caam A, Madej W, Thijssen E, Garcia de Vinuesa A, van den Berg W, Goumans MJ, ten Dijke P, Blaney Davidson E, van der Kraan PM (2016) Expression of TGF $\beta$-family signalling components in ageing cartilage: age-related loss of TGF $\beta$ and BMP receptors. Osteoarthr Cartil 24(7):1235-1245

19. van Beuningen HM, de Vries-van Melle ML, Vitters EL, Schreurs W, van den Berg WB, van Osch GJVM, van der Kraan PM (2014) Inhibition of TAK1 and/or JAK can rescue impaired chondrogenic differentiation of human mesenchymal stem cells in osteoarthritis-like conditions. Tissue Eng Part A 20(15-16):2243-2252

20. Maroudas A, Stockwell RA, Nachemson A, Urban J (1975) Factors involved in the nutrition of the human lumbar intervertebral disc: cellularity and diffusion of glucose in vitro. J Anat 120(Pt 1):113-130

21. Roughley PJ, Melching LI, Heathfield TF, Pearce RH, Mort JS (2006) The structure and degradation of aggrecan in human intervertebral disc. Eur Spine J 15(Suppl 3):326-332

22. Mwale F, Roughley P, Antoniou J (2004) Distinction between the extracellular matrix of the nucleus pulposus and hyaline cartilage: a requisite for tissue engineering of intervertebral disc. Eur Cells Mater 8:58-64

23. Alini M, Eisenstein S, Ito K, Little C, Kettler A, Masuda K, Melrose J, Ralphs J, Stokes I, Wilke H (2008) Are animal models useful for studying human disc disorders/degeneration? Eur Spine J 17(1):2-19

24. Gilson A, Dreger M, Urban J (2010) Differential expression level of cytokeratin 8 in cells of the bovine nucleus pulposus complicates the search for specific intervertebral disc cell markers. Arthritis Res Ther 12(1):R24

25. Errington RJ, Puustjarvi K, White IRF, Roberts S, Urban JPG (1998) Characterisation of cytoplasm-filled processes in cells of the intervertebral disc. J Anat 192(Pt 3):369-378

26. Kraus P, Lufkin T (2017) Implications for a stem cell regenerative medicine based approach to human intervertebral disk degeneration. Front Cell Dev Biol 5:17

27. Iu J, Santerre JP, Kandel RA (2014) Inner and outer annulus fibrosus cells exhibit differentiated phenotypes and yield changes in extracellular matrix protein composition in vitro on a polycarbonate urethane scaffold. Tissue Eng Part A 20(23-24):3261-3269

28. Reza AT, Nicoll SB (2008) Hydrostatic pressure differentially regulates outer and inner annulus fibrosus cell matrix production in 3D scaffolds. Ann Biomed Eng 36(2):204-213

29. Bruehlmann SB, Rattner JB, Matyas JR, Duncan NA (2002) Regional variations in the cellular matrix of the annulus fibrosus of the intervertebral disc. J Anat 201(2):159-171

30. Clarke LE, McConnell JC, Sherratt MJ, Derby B, Richardson SM, Hoyland JA (2014) Growth differentiation factor 6 and transforming growth factor-beta differentially mediate mesenchymal stem cell differentiation, composition and micromechanical properties of nucleus pulposus constructs. Arthritis Res Ther 16(2):R67 